\title{
Stereoselective Synthesis of the Lituarine Tricyclic Spiroacetal
}

\section{Jeremy Robertson,* Paul Meo, Jonathan W. P. Dallimore, Bryan Doyle and Christophe Hoarau}

\section{Supporting Information}

\section{2-Hydroxy-1,6-dioxaspiro[4.5]dec-3-ene (7)}

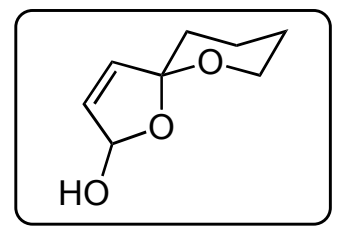

MCPBA (12.3 g, 70\% by weight, $50.0 \mathrm{mmol})$ was added in portions to a stirred solution of 2-(4hydroxybutyl)furan $^{1}(7.0 \mathrm{~g}, 50 \mathrm{mmol})$ in dichloromethane $(150 \mathrm{~mL})$ at $0{ }^{\circ} \mathrm{C}$. After $2 \mathrm{~h}$ at $0{ }^{\circ} \mathrm{C}$ the reaction mixture was poured into a solution consisting of saturated sodium hydrogen carbonate solution $(50 \mathrm{~mL})$ and sodium thiosulfate solution $(50 \mathrm{~mL})$; after shaking, the organic layer was run off and the aqueous layer extracted with chloroform $(3 \square 50 \mathrm{~mL})$. The combined organic portions were washed with brine $(50 \mathrm{~mL})$, dried $\left(\mathrm{Na}_{2} \mathrm{SO}_{4}\right)$, filtered, and concentrated in vacuo to give a residue that was purified by chromatography on silica gel (petrol/ether, 4:1 $\square$ ether); spirolactol 7 was obtained as a

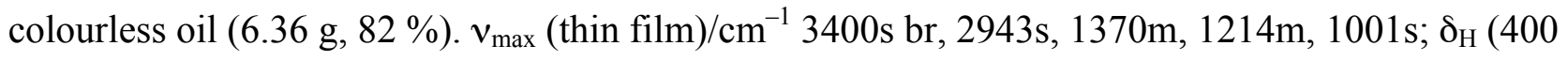
$\left.\mathrm{MHz}, \mathrm{CDCl}_{3}\right)$ 1.57-1.98 $\left(6 \mathrm{H}, \mathrm{m}, \mathrm{C}_{3} \mathrm{H}_{6}\right), 3.75-3.80(1 \mathrm{H}, \mathrm{m})$ and $3.97-4.09\left(1 \mathrm{H}, \mathrm{m}, \mathrm{CH}_{2} \mathrm{O}\right), 5.82(1 \mathrm{H}$, $\mathrm{m}, \mathrm{CHOH}), 6.03(1 \mathrm{H}, \mathrm{dd}, J 5.8,1.2 \mathrm{~Hz})$ and $6.07(1 \mathrm{H}, \mathrm{dd}, J 5.8,1.2 \mathrm{~Hz}, \mathrm{CH}=\mathrm{CH}) ; \square_{\mathrm{c}}(100 \mathrm{MHz}$, $\left.\mathrm{CDCl}_{3}\right)$ data for major diastereomer: $19.3\left(\mathrm{CH}_{2}\right), 24.8\left(\mathrm{CH}_{2}\right), 33.6(\mathrm{CH} 2), 63.4\left(\mathrm{CH}_{2}\right), 101.8(\mathrm{CH})$, $108.5(\mathrm{C}), 131.6(\mathrm{CH}), 135.2(\mathrm{CH})$; HRMS found 157.0860; $\mathrm{C}_{8} \mathrm{H}_{13} \mathrm{O}_{3}\left(\mathrm{MH}^{+}\right)$requires 157.0865.

(1) Sun, M.; Deng, Y.; Batyreva, E.; Sha, W.; Salomon, R. G. J. Org. Chem. 2002, 67, 3575-3584. 
1,6-Dioxaspiro[4.5]dec-3-en-2-one $(8)^{2}$

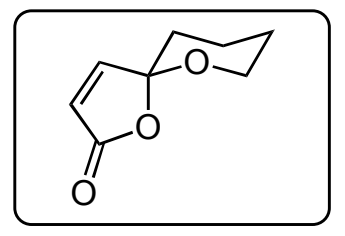

To a stirred suspension of pyridinium dichromate $(20.9 \mathrm{~g}, 55.6 \mathrm{mmol})$ in dimethylformamide $(80 \mathrm{~mL})$ at $0{ }^{\circ} \mathrm{C}$ under argon was added dropwise lactol $7(6.30 \mathrm{~g}, 40.4 \mathrm{mmol})$. The mixture was stirred at $0{ }^{\circ} \mathrm{C}$ for $4 \mathrm{~h}$ and was then diluted with water $(50 \mathrm{~mL})$, extracted with ether $(3 \square 50 \mathrm{~mL})$, and the combined extracts were dried $\left(\mathrm{Na}_{2} \mathrm{SO}_{4}\right)$, filtered, and concentrated in vacuo. The crude product was purified on activated alumina (petrol/ether, 4:1 $\square$ ether) to give spirobutenolide 8 as a colourless oil (4.65 g, 75

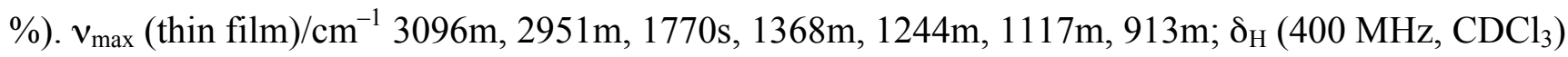
1.64-1.74 $(3 \mathrm{H}, \mathrm{m})$ and 1.79-1.92 $\left(3 \mathrm{H}, \mathrm{m}, \mathrm{C}_{3} \mathrm{H}_{6}\right), 3.86-3.91(1 \mathrm{H}, \mathrm{m})$ and 3.96-4.03 $\left(1 \mathrm{H}, \mathrm{m}, \mathrm{CH}_{2} \mathrm{O}\right)$, $6.08\left(1 \mathrm{H}, \mathrm{d}, J 5.6 \mathrm{~Hz},=\mathrm{CHCO}_{2}\right), 7.12\left(1 \mathrm{H}, \mathrm{d}, J 5.6 \mathrm{~Hz}, \mathrm{CH}=\mathrm{CHCO}_{2}\right)$; $\square_{\mathrm{c}}\left(100 \mathrm{MHz}, \mathrm{CDCl}_{3}\right) 18.9$ $\left(\mathrm{CH}_{2}\right), 24.0\left(\mathrm{CH}_{2}\right), 32.0\left(\mathrm{CH}_{2}\right), 65.0\left(\mathrm{CH}_{2}\right), 106.9(\mathrm{C}), 122.9(\mathrm{CH}), 154.3(\mathrm{CH}), 170.5(\mathrm{C})$; HRMS found 155.0701; $\mathrm{C}_{8} \mathrm{H}_{11} \mathrm{O}_{3}\left(\mathrm{MH}^{+}\right)$requires 155.0708.

\section{4-Methyl-1,6-dioxaspiro[4.5]decan-2-one (9)}

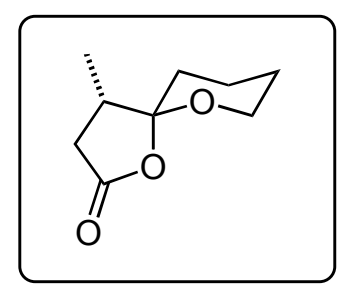

Butyllithium (5.80 mL, 2.5 M in hexanes, $14.5 \mathrm{mmol})$ was added dropwise to a stirred solution of tris(methanethio)methane $(1.94 \mathrm{~mL}, 14.6 \mathrm{mmol})$ in anhydrous tetrahydrofuran $(20 \mathrm{~mL})$ at $-78{ }^{\circ} \mathrm{C}$ under argon. After $0.5 \mathrm{~h}$, a solution of spirobutenolide $8(1.34 \mathrm{~g}, 8.70 \mathrm{mmol})$ in anhydrous tetrahydrofuran $(10 \mathrm{~mL})$ was added over $10 \mathrm{~min}$ and the mixture was stirred for $5 \mathrm{~h}$ at $-78^{\circ} \mathrm{C}$. The reaction was

(2) Fukuda, H.; Takeda, M.; Sato, Y.; Mitsunobu, O. Synthesis 1979, 368-370. 
quenched by pouring the mixture into a saturated solution of ammonium chloride $(50 \mathrm{~mL})$, which was then extracted with ether $(3 \square 40 \mathrm{~mL})$. The combined extracts were dried $\left(\mathrm{Na}_{2} \mathrm{SO}_{4}\right)$, filtered, and concentrated in vacuo to give a residue that was purified by chromatography on silica gel (petrol/ether, 4:1 $\square$ ether); the adduct was isolated as a colourless oil that solidified slowly on standing (1.57 $\mathrm{g}$, 59\%). $\square_{\max }\left(\right.$ thin film) $/ \mathrm{cm}^{-1} 2920 \mathrm{~m}, 1774 \mathrm{~m}, 1437 \mathrm{w}, 1215 \mathrm{w}, 918 \mathrm{w} ; \square_{\mathrm{H}}\left(400 \mathrm{MHz}, \mathrm{CDCl}_{3}\right) 1.54-1.60$ $(1 \mathrm{H}, \mathrm{m}), 1.63-1.75(1 \mathrm{H}, \mathrm{m}), 1.75-1.81(1 \mathrm{H}, \mathrm{m})$ and 1.82-1.91 $\left(1 \mathrm{H}, \mathrm{m}, \mathrm{C}_{2} \mathrm{H}_{4}\right), 2.21\left(9 \mathrm{H}, \mathrm{s}, \mathrm{C}(\mathrm{SMe})_{3}\right)$, 2.21-2.28 (1H, m) and 2.30-2.39 (1H, m, $\left.\mathrm{CH}_{2} \mathrm{C}(\mathrm{OR}) \mathrm{O}\right), 2.93\left(1 \mathrm{H}, \mathrm{dd}, J 10.1,8.4 \mathrm{~Hz}, \mathrm{CHC}(\mathrm{SMe})_{3}\right)$, $2.94(1 \mathrm{H}, \mathrm{dd}, J 20.1,8.4 \mathrm{~Hz})$ and $3.11\left(1 \mathrm{H}, \mathrm{dd}, J 20.1,10.1 \mathrm{~Hz}, \mathrm{CH}_{2} \mathrm{C}=\mathrm{O}\right), 3.77-3.82(1 \mathrm{H}, \mathrm{m})$ and 3.92-3.99 (1H, m, $\left.\mathrm{CH}_{2} \mathrm{O}\right)$; $\square_{\mathrm{c}}\left(100 \mathrm{MHz}, \mathrm{CDCl}_{3}\right)$ 14.6 $\left(\mathrm{CH}_{3}\right), 18.9\left(\mathrm{CH}_{2}\right), 24.5\left(\mathrm{CH}_{2}\right), 31.6\left(\mathrm{CH}_{2}\right), 33.8$ $\left(\mathrm{CH}_{2}\right), 55.6(\mathrm{CH}), 64.2\left(\mathrm{CH}_{2}\right), 69.5(\mathrm{C}), 109.9(\mathrm{C}), 174.1(\mathrm{C})$; HRMS found 261.0603; $\mathrm{C}_{11} \mathrm{H}_{17} \mathrm{O}_{3} \mathrm{~S}_{3}$ $\left(\mathrm{M}^{+}-\mathrm{SMe}\right)$ requires 261.0619. A sample of this adduct $(195 \mathrm{mg}, 0.63 \mathrm{mmol})$ was dissolved in tetrahydrofuran, stirred and Raney nickel $(10 \mathrm{~mL}, 50 \%$ slurry in water) was added. After $6 \mathrm{~h}$, the solvent was decanted, ether $(30 \mathrm{~mL})$ was added to the residue and the suspension stirred for $15 \mathrm{~min}$; this procedure was repeated twice more with ether $(2 \square 30 \mathrm{~mL})$. The combined decanted organic portions were washed once with water $(20 \mathrm{~mL})$ then were dried $\left(\mathrm{Na}_{2} \mathrm{SO}_{4}\right)$, filtered, and concentrated in vacuo to give the title compound (9) as a colourless oil (58 mg, 54\%). $\square_{\max }\left(\right.$ thin film) $/ \mathrm{cm}^{-1} 2948 \mathrm{~s}$, $1787 \mathrm{~s}, 1236 \mathrm{~m}, 1099 \mathrm{~m}, 895 \mathrm{~s}$; П $\mathrm{H}_{\mathrm{H}}\left(400 \mathrm{MHz}, \mathrm{CDCl}_{3}\right) 1.01(3 \mathrm{H}, \mathrm{d}, J 7.3 \mathrm{~Hz}, \mathrm{Me}), 1.57-1.68(3 \mathrm{H}, \mathrm{m})$ and 1.74-1.88 (3H, m, C $\left.{ }_{3} \mathrm{H}_{6}\right), 2.09(1 \mathrm{H}, \mathrm{dd}, J 17.3,2.3 \mathrm{~Hz}, \mathrm{CHHC}=\mathrm{O}), 2.32-2.41(1 \mathrm{H}, \mathrm{m}, \mathrm{CHMe}), 2.98$ $(1 \mathrm{H}, \mathrm{dd}, J 17.2,8.0 \mathrm{~Hz}, \mathrm{CHHC}=\mathrm{O}), 3.71-3.76(1 \mathrm{H}, \mathrm{m})$ and 3.86-3.93 $\left(1 \mathrm{H}, \mathrm{m}, \mathrm{CH}_{2} \mathrm{O}\right)$ a peak attributable to a minor diastereomer (1:19 ratio with major isomer) appears at $1.09(3 \mathrm{H}, \mathrm{d}, J 7.2 \mathrm{~Hz}$, Me); $\square_{\mathrm{c}}\left(100 \mathrm{MHz}, \mathrm{CDCl}_{3}\right) 15.3\left(\mathrm{CH}_{3}\right), 19.1\left(\mathrm{CH}_{2}\right), 24.5\left(\mathrm{CH}_{2}\right), 29.4\left(\mathrm{CH}_{2}\right), 36.8\left(\mathrm{CH}_{2}\right), 39.5(\mathrm{CH})$, $63.3\left(\mathrm{CH}_{2}\right), 109.4(\mathrm{C}), 176.4(\mathrm{C})$; HRMS found 171.1017; $\mathrm{C}_{9} \mathrm{H}_{15} \mathrm{O}_{3}\left(\mathrm{MH}^{+}\right)$requires 171.1021 . 


\section{(4R)-4-(tert-Butyldimethylsilanyloxy)-5-oxohexanal (12) ${ }^{3}$}

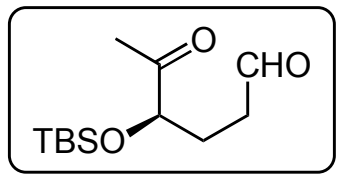

A solution of 2-methylcylclopentenone $(4.33 \mathrm{~g}, 45.1 \mathrm{mmol})$ in anhydrous dichloromethane $(86 \mathrm{~mL})$ was cooled to $-78{ }^{\circ} \mathrm{C}$ and allowed to stir for $10 \mathrm{~min}$. $(S)-\mathrm{CBS} \cdot \mathrm{BH}_{3}$ complex $(13.1 \mathrm{~g}, 45.0 \mathrm{mmol})$ was added in one portion and the reaction mixture stirred for $2 \mathrm{~h}$ at $-78^{\circ} \mathrm{C}$. Methanol $(50 \mathrm{~mL})$ was added and the reaction mixture stirred for a further $15 \mathrm{~min}$. After careful evaporation of the solvent, saturated sodium hydrogen carbonate solution $(100 \mathrm{~mL})$ was added and the mixture extracted with ether $(4 \square 50$ $\mathrm{mL}$ ). The combined organic phases were washed successively with saturated ammonium chloride solution $(2 \square 100 \mathrm{~mL})$, and brine $(100 \mathrm{~mL})$, then dried $\left(\mathrm{MgSO}_{4}\right)$, filtered, and the solvent removed in vacuo. [The ${ }^{19}$ F NMR spectrum of the $(R)$-Mosher's ester derivative of $( \pm)$-10 exhibited resonances at $\square$ -71.46 and -71.51 p.p.m. in equal ratio; in the analogous spectrum of $(+)-\mathbf{1 0}$, obtained by this procedure, the two resonances integrated respectively to a 32:1 ratio $(e e=94 \%)$.] The crude product (3.97 $\mathrm{g}, 90 \%)$ was dissolved in anhydrous dimethylformamide $(45 \mathrm{~mL})$ and cooled to $0{ }^{\circ} \mathrm{C}$. Imidazole (3.31 g, $48.6 \mathrm{mmol})$ and tert-butyldimethylsilyl chloride (7.32 g, $48.6 \mathrm{mmol})$ were added and the reaction stirred at room temperature for $8 \mathrm{~h}$. The reaction was quenched by the addition of water (45 $\mathrm{mL})$ and extracted with ether $(3 \square 40 \mathrm{~mL})$; the combined organic fractions were washed with brine, dried $\left(\mathrm{MgSO}_{4}\right)$, filtered, and the solvent removed in vacuo. The residue was subjected to column chromatography using silica gel (petrol/ether, 20:1) to afford a yellow oil $(7.50 \mathrm{~g}, 87 \%)$ which was dissolved in dichloromethane $(150 \mathrm{~mL})$ and cooled to $-78{ }^{\circ} \mathrm{C}$; ozone was then bubbled through the reaction mixture and, after $40 \mathrm{~min}$, a persistent blue colour was observed and the mixture was purged with argon. Triphenylphosphine $(16.5 \mathrm{~g}, 63 \mathrm{mmol})$ was added and the reaction stirred at $-78^{\circ} \mathrm{C}$ for $4 \mathrm{~h}$.

(3) ( \pm )-12 is known: Uenishi, J.-i.; Masuda, S.; Wakabayashi, S. Tetrahedron Lett. 1991, 32, 5097-5100. 
The solvent was removed in vacuo and the residue was subjected to column chromatography using

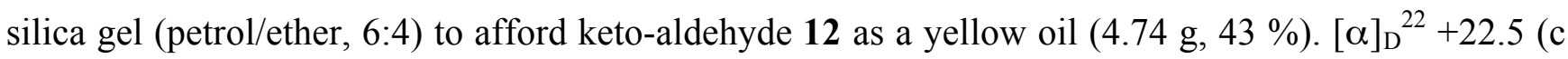
$\left.1.0, \mathrm{CHCl}_{3}\right)$; $\square_{\max }\left(\right.$ thin film) $/ \mathrm{cm}^{-1} 2956 \mathrm{~s}, 2931 \mathrm{~s}, 2858 \mathrm{~s}, 1726 \mathrm{~s}, 1354 \mathrm{~m}, 1255 \mathrm{~s}, 1117 \mathrm{~s}, 1006 \mathrm{~m}, 839 \mathrm{~s}$, $779 \mathrm{~s}$; $\square_{\mathrm{H}}\left(400 \mathrm{MHz}, \mathrm{CDCl}_{3}\right) 0.04\left(6 \mathrm{H}, \mathrm{s}, \mathrm{Me}_{2} \mathrm{Si}\right), 0.90(9 \mathrm{H}, \mathrm{s}, t-\mathrm{Bu}), 1.90-1.96\left(2 \mathrm{H}, \mathrm{m}, \mathrm{CH}_{2} \mathrm{CH}_{2} \mathrm{CHO}\right)$, 2.15 (3H, s, MeCO), 2.46-2.51 (2H, m, $\left.\mathrm{CH}_{2} \mathrm{CHO}\right), 4.03$ (1H, apparent t, J 6.1 Hz, CHOTBS), 9.74 $(1 \mathrm{H}, \mathrm{t}, J 1.0 \mathrm{~Hz}, \mathrm{CHO})$; $\square_{\mathrm{c}}\left(100 \mathrm{MHz}, \mathrm{CDCl}_{3}\right)-5.1\left(\mathrm{CH}_{3}\right), 18.00(\mathrm{C}), 25.5\left(\mathrm{CH}_{3}\right), 25.6\left(\mathrm{CH}_{3}\right), 26.7$ $\left(\mathrm{CH}_{2}\right), 39.0\left(\mathrm{CH}_{2}\right), 77.4(\mathrm{CH}), 201.1(\mathrm{CH})$; HRMS found 245.1579; $\mathrm{C}_{12} \mathrm{H}_{25} \mathrm{O}_{3} \mathrm{Si}\left(\mathrm{MH}^{+}\right)$requires 245.1573.

\section{(6R)-(E)-7-oxo-6-(tert-butyldimethylsilanyloxy)-oct-2-enoic acid ethyl ester (13)}

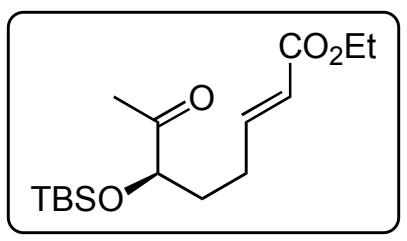

A solution of triethylphosphonoacetate $(0.89 \mathrm{~mL}, 4.5 \mathrm{mmol})$ in tetrahydrofuran $(30 \mathrm{ml})$ was cooled to $-78^{\circ} \mathrm{C}$ and butyllithium $(3.1 \mathrm{~mL}, 1.6 \mathrm{M}$ in hexanes, $5.0 \mathrm{mmol})$ was added dropwise. After $30 \mathrm{~min}$ at $-78{ }^{\circ} \mathrm{C}$ a solution of keto-aldehyde $12(1.0 \mathrm{~g}, 4.1 \mathrm{mmol})$ was added dropwise. The resulting mixture was stirred for $30 \mathrm{~min}$ and was then warmed up to $0{ }^{\circ} \mathrm{C}$ for an additional $90 \mathrm{~min}$. The reaction was quenched with saturated ammonium chloride solution $(10 \mathrm{~mL})$ and extracted with ether $(3 \square 15 \mathrm{ml})$. The combined organic extracts were dried $\left(\mathrm{MgSO}_{4}\right)$ and the solvents were removed in vacuo. Purification by flash column chromatography (petrol/ether, 6:4) gave ester $\mathbf{1 3}$ as a limpid oil (1.13 g,

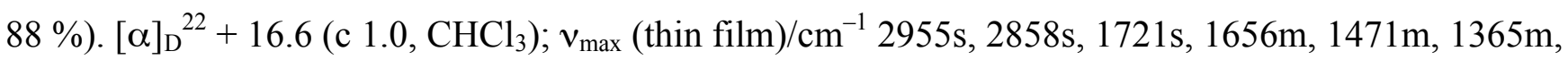
1260s, $1114 \mathrm{~m}, 1044 \mathrm{~m}, 838 \mathrm{~s}, 779 \mathrm{~s} ; \square_{\mathrm{H}}\left(400 \mathrm{MHz}, \mathrm{CDCl}_{3}\right) 0.05$ and $0.06\left(2 \square 3 \mathrm{H}, 2 \square \mathrm{s}, \mathrm{Me}_{2} \mathrm{Si}\right), 0.91$ $(9 \mathrm{H}, \mathrm{s}, t-\mathrm{Bu}), 1.27\left(3 \mathrm{H}, \mathrm{t}, J 7.2 \mathrm{~Hz}, \mathrm{OCH}_{2} \mathrm{CH}_{3}\right), 1.67-1.85\left(2 \mathrm{H}, \mathrm{m}, \mathrm{CH}_{2} \mathrm{CH}(\mathrm{OTBS})\right), 2.16(3 \mathrm{H}, \mathrm{s}$, MeCO), 2.21-2.28 (2H, m, $\left.\mathrm{CH}_{2} \mathrm{CH}=\right), 4.01$ (1H, dd, J 6.6, 5.0 Hz, CH(OTBS)), 4.17 (2H, q, J 7.2 Hz, 
$\left.\mathrm{OCH}_{2} \mathrm{CH}_{3}\right), 5.82\left(1 \mathrm{H}, \mathrm{dt}, J 15.6,1.6 \mathrm{~Hz},=\mathrm{CHCO}_{2}\right), 6.92\left(1 \mathrm{H}, \mathrm{dt}, J 15.6,6.9 \mathrm{~Hz}, \mathrm{CH}_{2} \mathrm{CH}=\right)$; $\square_{\mathrm{c}}(100$ $\left.\mathrm{MHz}, \mathrm{CDCl}_{3}\right)-5.0\left(\mathrm{CH}_{3}\right), 14.2\left(\mathrm{CH}_{3}\right), 18.0(\mathrm{C}), 25.4\left(\mathrm{CH}_{3}\right), 25.7\left(\mathrm{CH}_{3}\right), 27.3\left(\mathrm{CH}_{2}\right), 32.9\left(\mathrm{CH}_{2}\right), 60.2$ $\left(\mathrm{CH}_{2}\right), 78.0(\mathrm{CH}), 121.9(\mathrm{CH}), 147.7(\mathrm{CH}), 166.4(\mathrm{C}), 211.8(\mathrm{C})$; HRMS found 315.1996; $\mathrm{C}_{16} \mathrm{H}_{31}, \mathrm{O}_{4} \mathrm{Si}$ $\left(\mathrm{MH}^{+}\right)$requires 315.1992.

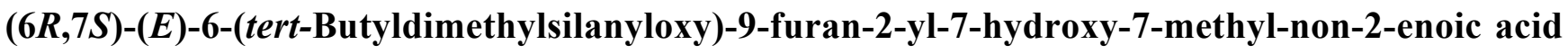
ethyl ester (16)

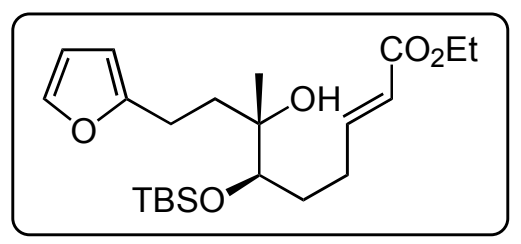

A degassed solution of 2-(2-bromoethyl)furan $(1.15 \mathrm{~g}, 6.57 \mathrm{mmol})$ in anhydrous, degassed tetrahydrofuran $(2 \mathrm{~mL})$ was added dropwise to a suspension of magnesium turnings (175 $\mathrm{mg}, 7.3$ $\mathrm{mmol})$ and one crystal of iodine in anhydrous, degassed tetrahydrofuran $(2 \mathrm{~mL})$. The reaction mixture was allowed to stir for $2 \mathrm{~h}$ until no further magnesium was consumed. The solution was added dropwise to a stirring solution of keto-ester $13(290 \mathrm{mg}, 0.92 \mathrm{mmol})$ in anhydrous tetrahydrofuran (5 $\mathrm{mL})$ at $-40{ }^{\circ} \mathrm{C}$. Stirring was continued for $90 \mathrm{~min}$ then an ice-water slurry $(10 \mathrm{~mL})$ was added. The resulting mixture was extracted with ether $(4 \square 10 \mathrm{~mL})$ and the combined organic extracts were washed with brine $(3 \square 20 \mathrm{~mL})$, then dried $\left(\mathrm{MgSO}_{4}\right)$, filtered, and the solvent removed in vacuo. The residue was subjected to column chromatography using silica gel (petrol/ether/dichloromethane, 5:1:1) to afford alcohol $16(171 \mathrm{mg}, 45 \%)$ as a colourless oil. [ $]_{\mathrm{D}}^{22}+2.6\left(\mathrm{c} 1.0, \mathrm{CHCl}_{3}\right)$; $\square_{\max } / \mathrm{cm}^{-1}$ (thin film) 3509m, 2955s, 2857s, 1720s, 1654m, 1596w, 1508w, 1097s, 836s; 口н (400 MHz, $\left.\mathrm{CDCl}_{3}\right) 0.11(3 \mathrm{H}, \mathrm{s}$, $\mathrm{Me}), 0.12(3 \mathrm{H}, \mathrm{s}, \mathrm{Me}), 0.90(9 \mathrm{H}, \mathrm{s}, t-\mathrm{Bu}), 1.14(3 \mathrm{H}, \mathrm{s}, \mathrm{Me}), 1.26\left(3 \mathrm{H}, \mathrm{t}, J 7.0 \mathrm{~Hz}, \mathrm{OCH}_{2} \mathrm{CH}_{3}\right)$, 1.54-1.62 (1H, m, $\left.\mathrm{CHHCH}_{2} \mathrm{CH}=\right), 1.63-1.74\left(2 \mathrm{H}, \mathrm{m}, \mathrm{CHHCH}_{2} \mathrm{CH}=\right.$ and $\left.\mathrm{CHHC}(\mathrm{OH})\right), 1.89(1 \mathrm{H}$, ddd, $J$ 13.6, 11.3, $5.2 \mathrm{~Hz}, \mathrm{CHHC}(\mathrm{OH})), 2.18-2.26(1 \mathrm{H}, \mathrm{m})$ and 2.37-2.47 $\left(1 \mathrm{H}, \mathrm{m}, \mathrm{CH}_{2} \mathrm{CH}=\right), 2.66(1 \mathrm{H}$, ddd, 
$J$ 15.2, 11.8, 5.2 Hz) and $2.79\left(1 \mathrm{H}, \mathrm{ddd}, J 15.2,11.3,4.4 \mathrm{~Hz}, \mathrm{CH}_{2} \mathrm{Fn}\right), 3.51(1 \mathrm{H}, \mathrm{dd}, J 6.8,3.8 \mathrm{~Hz}$, CHOTBS), $4.16\left(2 \mathrm{H}, \mathrm{q}, J 7.0 \mathrm{~Hz}, \mathrm{OCH}_{2}\right), 5.81\left(1 \mathrm{H}, \mathrm{dt}, J 15.6,1.6 \mathrm{~Hz},=\mathrm{CHCO}_{2} \mathrm{Et}\right), 5.97(1 \mathrm{H}, \mathrm{dd}, J$ 2.4, 1.2 Hz, Fn), $6.25(1 \mathrm{H}, \mathrm{dd}, J 3.2,2.4 \mathrm{~Hz}, \mathrm{Fn}), 6.93\left(1 \mathrm{H}, \mathrm{dt}, J 15.6,6.8 \mathrm{~Hz}, \mathrm{CH}=\mathrm{CHCO}_{2} \mathrm{Et}\right), 7.28$ $(1 \mathrm{H}, \mathrm{dd}, J 3.2,1.2 \mathrm{~Hz}, \mathrm{Fn})$; $\square_{\mathrm{c}}\left(100 \mathrm{MHz}, \mathrm{CDCl}_{3}\right)-4.3\left(\mathrm{CH}_{3}\right),-3.9\left(\mathrm{CH}_{3}\right), 14.2\left(\mathrm{CH}_{3}\right), 18.2(\mathrm{C}), 22.3$ $\left(\mathrm{CH}_{2}\right), 23.4\left(\mathrm{CH}_{3}\right), 25.6\left(\mathrm{CMe}_{3}\right), 29.1\left(\mathrm{CH}_{2}\right), 31.5\left(\mathrm{CH}_{2}\right), 34.7\left(\mathrm{CH}_{2}\right), 60.1\left(\mathrm{CH}_{2}\right), 74.3(\mathrm{C}), 78.8(\mathrm{CH})$, $104.4(\mathrm{CH}), 110.0(\mathrm{CH}), 121.4(\mathrm{CH}), 140.7(\mathrm{CH}), 148.6(\mathrm{CH}), 156.3(\mathrm{C}), 166.5(\mathrm{C})$; HRMS found 433.2402; $\mathrm{C}_{22} \mathrm{H}_{38} \mathrm{O}_{5} \mathrm{NaSi}\left(\mathrm{MNa}^{+}\right)$requires 433.2386.

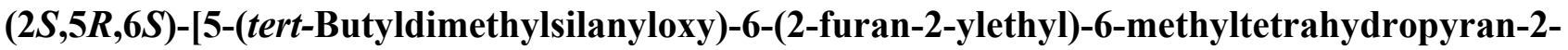
yl]acetic acid ethyl ester (17)

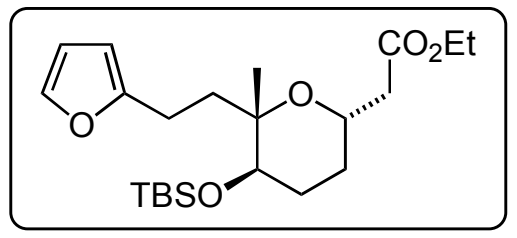

To a stirring solution of hexamethyldisilazane $(0.1 \mathrm{~mL}, 0.46 \mathrm{mmol})$ in anhydrous tetrahydrofuran $(2$ $\mathrm{mL})$ at $-78{ }^{\circ} \mathrm{C}$ was added butyllithium $(0.29 \mathrm{~mL}, 0.46 \mathrm{mmol}, 1.6 \mathrm{M}$ in hexanes $)$. The reaction mixture was allowed to stir for $30 \mathrm{~min}$ and then added to a stirring solution of alcohol 16 (171 $\mathrm{mg}, 0.42 \mathrm{mmol})$ in anhydrous tetrahydrofuran $(5 \mathrm{~mL})$ at $-78^{\circ} \mathrm{C}$. The reaction mixture was stirred for $3 \mathrm{~h}$ with gradual warming to $-40{ }^{\circ} \mathrm{C}$. The reaction was quenched by the addition of saturated ammonium chloride solution $(10 \mathrm{~mL})$ and extracted with ether $(4 \square 10 \mathrm{~mL})$, the combined organic extracts were washed with brine $(15 \mathrm{~mL})$, dried $\left(\mathrm{MgSO}_{4}\right)$, filtered, and the solvent removed in vacuo. The residue was subjected to column chromatography using silica gel (petrol/ether/dichloromethane, 5:1:1) to afford the

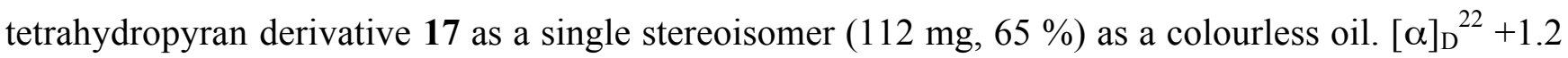
$\left(c 1.0, \mathrm{CHCl}_{3}\right) ; \square_{\max } / \mathrm{cm}^{-1}$ (thin film) 2929s, 2862s, 1732s, 1508s, 1377m, 1259s, 1098s, 837m, 776m; $\square_{\mathrm{H}}\left(400 \mathrm{MHz}, \mathrm{CDCl}_{3}\right) 0.05\left(6 \mathrm{H}, \mathrm{s}, \mathrm{Me}_{2} \mathrm{Si}\right), 0.87(9 \mathrm{H}, \mathrm{s}, t-\mathrm{Bu}), 1.16(3 \mathrm{H}, \mathrm{s}, \mathrm{Me}), 1.27(3 \mathrm{H}, \mathrm{t}, J 7.0 \mathrm{~Hz}$, 
$\left.\mathrm{OCH}_{2} \mathrm{CH}_{3}\right), 1.24-1.30$ (2H, m, $\left.\mathrm{CH}_{2} \mathrm{CHO}\right), 1.65-1.77$ (3H, m, CH $\left.2 \mathrm{CH}(\mathrm{OTBS}), \mathrm{CHHCH}_{2} \mathrm{Fn}\right), 1.94$ (1H, ddd, $\left.J 13.2,11.2,5.6 \mathrm{~Hz}, \mathrm{CHHCH}_{2} \mathrm{Fn}\right), 2.34(1 \mathrm{H}$, dd, $J 14.9,5.0 \mathrm{~Hz})$ and $2.43(1 \mathrm{H}, \mathrm{dd}, J 14.9,7.6 \mathrm{~Hz}$, $\left.\mathrm{CH}_{2} \mathrm{CO}_{2} \mathrm{Et}\right), 2.65-2.72\left(2 \mathrm{H}, \mathrm{m}, \mathrm{CH}_{2} \mathrm{Fn}\right), 3.47$ (1H, dd, $J$ 10.6, $\left.5.0 \mathrm{~Hz}, \mathrm{CH}(\mathrm{OTBS})\right), 3.95$ (1H, dddd, $J$ 12.0, 7.6, 5.0, $\left.1.8 \mathrm{~Hz}, \mathrm{CHCH}_{2} \mathrm{CO}_{2} \mathrm{Et}\right), 4.12-4.19\left(2 \mathrm{H}, \mathrm{m}, \mathrm{OCH}_{2} \mathrm{CH}_{3}\right), 5.93(1 \mathrm{H}, \mathrm{dd}, J 3.2,0.8 \mathrm{~Hz}, \mathrm{Fn})$, $6.26(1 \mathrm{H}, \mathrm{dd}, J 3.2,1.6 \mathrm{~Hz}, \mathrm{Fn}), 7.28(1 \mathrm{H}, \mathrm{dd}, J 1.2,0.8 \mathrm{~Hz}, \mathrm{Fn})$; $\square_{\mathrm{c}}\left(100 \mathrm{MHz}, \mathrm{CDCl}_{3}\right)-4.9\left(\mathrm{CH}_{3}\right)$, -3.9 $\left(\mathrm{CH}_{3}\right), 14.3\left(\mathrm{CH}_{3}\right), 14.9(\mathrm{Me}), 17.8(\mathrm{C}), 21.2\left(\mathrm{CH}_{2}\right), 25.7\left(\mathrm{CH}_{3}\right), 28.6\left(\mathrm{CH}_{2}\right), 30.9\left(\mathrm{CH}_{2}\right), 38.5$ $\left(\mathrm{CH}_{2}\right), 41.5\left(\mathrm{CH}_{2}\right), 60.3\left(\mathrm{CH}_{2}\right), 66.5(\mathrm{CH}), 72.9(\mathrm{CH}), 76.6(\mathrm{C}), 104.0(\mathrm{CH}), 110.0(\mathrm{CH}), 140.5(\mathrm{CH})$, 152.0 (C), 171.5 (C); HRMS found 433.2402; $\mathrm{C}_{22} \mathrm{H}_{38} \mathrm{O}_{5} \mathrm{NaSi}\left(\mathrm{MNa}^{+}\right)$requires 433.2386.

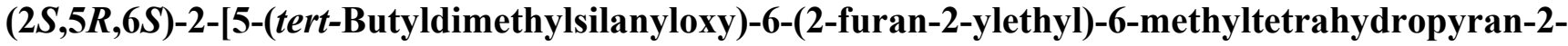
yl]ethanol (18)

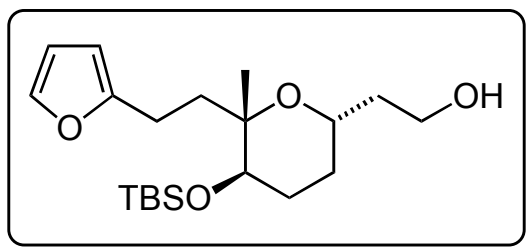

Diisobutylaluminium hydride $(1.1 \mathrm{~mL}, 1.1 \mathrm{mmol}, 1 \mathrm{M}$ in hexanes) was added dropwise to a stirring solution of ester $17(150 \mathrm{mg}, 0.37 \mathrm{mmol})$ in anhydrous tetrahydrofuran $(15 \mathrm{~mL})$ at $0{ }^{\circ} \mathrm{C}$. Once the reaction was shown to be complete by TLC the reaction mixture was quenched by the addition of $2 \mathrm{M}$ potassium sodium tartrate solution $(15 \mathrm{~mL})$ and extracted with ether $(2 \square 15 \mathrm{~mL})$. The combined organic extracts were washed with brine $(10 \mathrm{~mL})$, dried $\left(\mathrm{MgSO}_{4}\right)$, filtered, and the solvent removed in vacuo to afford the primary alcohol $18(114 \mathrm{mg}, 85 \%)$ as a yellow oil which required no further purification. $[\square]_{\mathrm{D}}^{22}-0.9\left(c 1.0, \mathrm{CHCl}_{3}\right)$; $\square_{\max } / \mathrm{cm}^{-1}$ (thin film) $3443 \mathrm{~m} \mathrm{br}, 2932 \mathrm{~s}, 2857 \mathrm{~s}, 1472 \mathrm{~m}, 1428 \mathrm{~m}$, $1389 \mathrm{w}, 1111 \mathrm{~s}, 736 \mathrm{~m}, 703 \mathrm{~s} ; \square_{\mathrm{H}}\left(400 \mathrm{MHz}, \mathrm{CDCl}_{3}\right) 0.058(3 \mathrm{H}, \mathrm{s}, \mathrm{Me}), 0.061(3 \mathrm{H}, \mathrm{s}, \mathrm{Me}), 0.87(9 \mathrm{H}, \mathrm{s}, t-$ $\mathrm{Bu}), 1.19(3 \mathrm{H}, \mathrm{s}, \mathrm{Me}), 1.35-1.42\left(1 \mathrm{H}, \mathrm{m}, \mathrm{CHHCH}_{2} \mathrm{CH}(\mathrm{OTBS})\right), 1.61-1.76(5 \mathrm{H}, \mathrm{m}$, $\left.\mathrm{CHHCH}_{2} \mathrm{CH}(\mathrm{OTBS}), \mathrm{CH}_{2} \mathrm{CH}_{2} \mathrm{OH}\right), 1.81(1 \mathrm{H}, \mathrm{ddd}, J 13.8,11.2,5.6 \mathrm{~Hz})$ and $1.95(1 \mathrm{H}, \mathrm{ddd}, J 13.8$, 
10.8, 6.4 Hz, $\left.\mathrm{CH}_{2} \mathrm{CH}_{2} \mathrm{Fn}\right), 2.63-2.75\left(2 \mathrm{H}, \mathrm{m}, \mathrm{CH}_{2} \mathrm{Fn}\right), 3.51(1 \mathrm{H}, \mathrm{dd}, J 10.8,4.8 \mathrm{~Hz}, \mathrm{CH}(\mathrm{OTBS}))$, 3.74-3.79 (3H, m, $\left.\mathrm{CHCH}_{2} \mathrm{CH}_{2} \mathrm{OH}\right), 5.94(1 \mathrm{H}, \mathrm{dd}, J 3.0,0.8 \mathrm{~Hz}, \mathrm{Fn}), 6.26(1 \mathrm{H}, \mathrm{dd}, J 3.0,1.8 \mathrm{~Hz}, \mathrm{Fn})$, $7.28(1 \mathrm{H}, \mathrm{dd}, J 1.8,0.8 \mathrm{~Hz}, \mathrm{Fn})$; $\mathrm{C}_{\mathrm{c}}\left(100 \mathrm{MHz}, \mathrm{CDCl}_{3}\right)-4.9\left(\mathrm{CH}_{3}\right),-3.9\left(\mathrm{CH}_{3}\right), 15.5\left(\mathrm{CH}_{3}\right), 17.8(\mathrm{C})$, $21.5\left(\mathrm{CH}_{2}\right), 25.7\left(\mathrm{CH}_{3}\right), 28.6\left(\mathrm{CH}_{2}\right), 31.3\left(\mathrm{CH}_{2}\right), 37.5\left(\mathrm{CH}_{2}\right), 38.3\left(\mathrm{CH}_{2}\right), 61.7\left(\mathrm{CH}_{2}\right), 70.5(\mathrm{CH}), 72.3$ $(\mathrm{CH}), 77.1(\mathrm{C}), 104.3(\mathrm{CH}), 110.0(\mathrm{CH}), 140.7(\mathrm{CH}), 156.4(\mathrm{C})$; HRMS found 369.2461; $\mathrm{C}_{20} \mathrm{H}_{37} \mathrm{O}_{4} \mathrm{Si}$ $\left(\mathrm{MH}^{+}\right)$requires 369.2461.

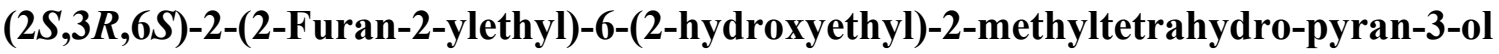

\section{(not numbered)}

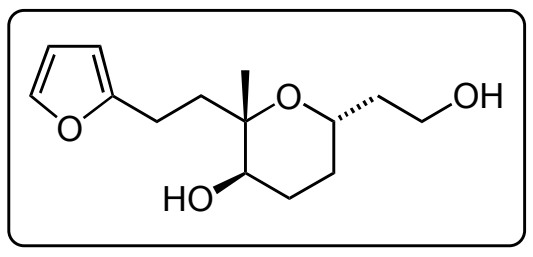

Fluorosilicic acid $(0.028 \mathrm{~mL}, 0.06 \mathrm{mmol}, 25 \% \mathrm{wt})$ was added to a stirring solution of silyl ether 18 $(110 \mathrm{mg}, 0.30 \mathrm{mmol})$ in acetonitrile $(5 \mathrm{~mL})$ at $0{ }^{\circ} \mathrm{C}$. The reaction mixture was allowed to stir for $5 \mathrm{~h}$ with gradual warming to room temperature. The reaction was quenched by the addition of saturated sodium hydrogen carbonate solution $(5 \mathrm{~mL})$ and the mixture extracted with ethyl acetate $(5 \square 10 \mathrm{~mL})$. The combined organic extracts were washed with brine $(10 \mathrm{~mL})$, dried $\left(\mathrm{MgSO}_{4}\right)$, filtered, and the solvent removed in vacuo. No further purification was required and the title compound was obtained as

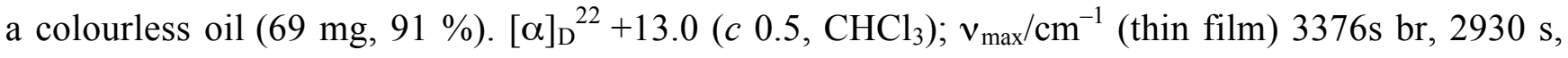
2857s, 1723w, 1597w, 1508m, 1442m, 1260m, 1080s, 801m; Пн (400 MHz, CDCl 3$) 1.24(3 \mathrm{H}, \mathrm{s}, \mathrm{Me})$, 1.38-1.48 (1H, m, CHHCH $2 \mathrm{CH}(\mathrm{OH})), 1.61-1.72$ (4H, m, CHHCHHCH(OH), $\left.\mathrm{CH}_{2} \mathrm{CH}_{2} \mathrm{OH}\right), 1.82-1.94$ $\left(2 \mathrm{H}, \mathrm{m}, \mathrm{CHHCH}_{2} \mathrm{Fn}, \mathrm{CHHCH}(\mathrm{OH})\right), 1.97-2.05$ (1H, m, $\left.\mathrm{CHHCH}_{2} \mathrm{Fn}\right), 2.70-2.78\left(2 \mathrm{H}, \mathrm{m}, \mathrm{CH}{ }_{2} \mathrm{Fn}\right)$, $3.53(1 \mathrm{H}, \mathrm{dd}, J 11.0,5.0 \mathrm{~Hz}, \mathrm{CH}(\mathrm{OH})), 3.74-3.77\left(3 \mathrm{H}, \mathrm{m}, \mathrm{CHCH}_{2} \mathbf{C H}_{2} \mathrm{OH}\right), 5.99(1 \mathrm{H}, \mathrm{dd}, J 3.2,0.8$ Hz, Fn), $6.27(1 \mathrm{H}, \mathrm{dd}, J 3.2,1.6 \mathrm{~Hz}, \mathrm{Fn}), 7.29(1 \mathrm{H}, \mathrm{dd}, J 1.6,0.8 \mathrm{~Hz}, \mathrm{Fn})$; 口c $\left(100 \mathrm{MHz}, \mathrm{CDCl}_{3}\right) 15.2$ 
$\left(\mathrm{CH}_{3}\right), 21.5\left(\mathrm{CH}_{2}\right), 28.1\left(\mathrm{CH}_{2}\right), 31.5\left(\mathrm{CH}_{2}\right), 37.5\left(\mathrm{CH}_{2}\right), 38.4\left(\mathrm{CH}_{2}\right), 61.6\left(\mathrm{CH}_{2}\right), 70.5(\mathrm{CH}), 71.8(\mathrm{CH})$, $76.7(\mathrm{C}), 104.5(\mathrm{CH}), 110.1(\mathrm{CH}), 140.7(\mathrm{CH}), 156.2(\mathrm{C})$; HRMS found 255.1592; $\mathrm{C}_{14} \mathrm{H}_{23} \mathrm{O}_{4}\left(\mathrm{MH}^{+}\right)$ requires 255.1596 .

(2S,3R,6S)-6-[2-(tert-Butyldiphenylsilanyloxy)ethyl]-2-(2-furan-2-ylethyl)-2-methyltetrahydropyran-3-ol (19)

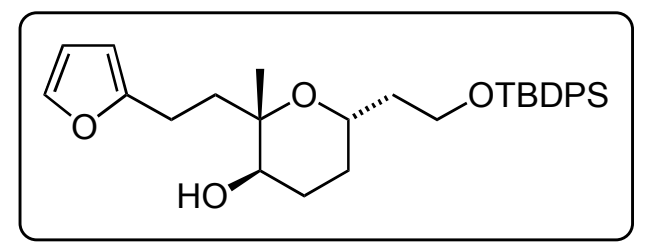

To a solution of $(2 S, 3 R, 6 S)$-2-(2-furan-2-ylethyl)-6-(2-hydroxyethyl)-2-methyltetrahydro-pyran-3-ol (50 $\mathrm{mg}, 0.20 \mathrm{mmol})$ in anhydrous dimethylformamide $(5 \mathrm{~mL})$ at room temperature was added imidazole (16.3 mg, $0.24 \mathrm{mmol})$ and tert-butyldiphenylsilyl chloride $(0.06 \mathrm{~mL}, 0.24 \mathrm{mmol})$. The reaction mixture was allowed to stir for $4 \mathrm{~h}$ and then the reaction was quenched by the addition of saturated ammonium chloride solution $(10 \mathrm{~mL})$ and extracted with ether $(4 \square 10 \mathrm{~mL})$. The combined organic extracts were washed with brine $(10 \mathrm{~mL})$, dried $\left(\mathrm{MgSO}_{4}\right)$, filtered, and the solvent removed in vacuo to afford mono-silyl ether $19(94 \mathrm{mg}, 97 \%$ ) as a yellow oil, sufficiently pure to use directly in the next reaction. $[\square]_{\mathrm{D}}^{22}+26.3\left(c 0.875, \mathrm{CHCl}_{3}\right)$; $\square$ max $/ \mathrm{cm}^{-1}$ (thin film) $3406 \mathrm{~m} \mathrm{br}, 3071 \mathrm{w}, 2932 \mathrm{~s}, 2858 \mathrm{~s}$, $1592 \mathrm{w}, 1508 \mathrm{~m}, 1472 \mathrm{~m}, 1428 \mathrm{w}, 1261 \mathrm{~m}, 1111 \mathrm{~s}, 823 \mathrm{~m}, 703 \mathrm{~s} ; \square_{\mathrm{H}}\left(400 \mathrm{MHz}, \mathrm{CDCl}_{3}\right) 1.06(9 \mathrm{H}, \mathrm{s}, t-\mathrm{Bu})$, $1.18(3 \mathrm{H}, \mathrm{s}, \mathrm{Me}), 1.24-1.36\left(1 \mathrm{H}, \mathrm{m}, \mathrm{CHHCH}_{2} \mathrm{CH}(\mathrm{OH})\right), 1.60-1.70(4 \mathrm{H}, \mathrm{m}, \mathrm{CHHCHHCH}(\mathrm{OH})$, $\left.\mathrm{CH}_{2} \mathrm{CH}_{2} \mathrm{OTBDPS}\right), 1.77-1.84$ (2H, m, $\left.\mathrm{CHHCH}_{2} \mathrm{Fn}, \mathrm{CHHCH}(\mathrm{OH})\right), 2.00$ (1H, apparent td, $J$ 12.6, 5.2 $\left.\mathrm{Hz}, \mathrm{CHHCH}_{2} \mathrm{Fn}\right), 2.62-2.80\left(2 \mathrm{H}, \mathrm{m}, \mathrm{CH}_{2} \mathrm{Fn}\right), 3.49(1 \mathrm{H}, \mathrm{dd}, J$ 11.2, $4.8 \mathrm{~Hz}, \mathrm{CH}(\mathrm{OH})), 3.67-3.76(2 \mathrm{H}$, m, $\mathrm{CHCH}_{2} \mathrm{CHHOTBDPS}$ ), 3.79-3.85 (1H, m, CHHOTBDPS), 5.96 (1H, dd, J 3.2, 0.8 Hz, Fn), 6.28 (1H, dd, J 3.0, 1.6 Hz, Fn), 7.29 (1H, dd, J 1.6, 0.8 Hz, Fn), 7.36-7.44 (6H, m, Ph), 7.66-7.70 (4H, m, $\mathrm{Ph})$; $\mathrm{C} \mathrm{c}\left(100 \mathrm{MHz}, \mathrm{CDCl}_{3}\right) 15.2\left(\mathrm{CH}_{3}\right), 21.8\left(\mathrm{CH}_{2}\right), 27.3\left(\mathrm{CH}_{3}\right), 29.0\left(\mathrm{CH}_{2}\right), 32.2\left(\mathrm{CH}_{2}\right), 39.2\left(\mathrm{CH}_{2}\right)$, 
$39.5\left(\mathrm{CH}_{2}\right), 60.8\left(\mathrm{CH}_{2}\right), 66.2(\mathrm{CH}), 73.4(\mathrm{CH}), 76.1(\mathrm{C}), 104.7(\mathrm{CH}), 110.5(\mathrm{CH}), 128.0(\mathrm{CH}), 130.0$ (CH), $136.0(\mathrm{CH}), 142.9(\mathrm{C}), 156.8(\mathrm{C})$ (one furan $\mathrm{CH}$ resonance obscured); HRMS found 493.2757; $\mathrm{C}_{30} \mathrm{H}_{41} \mathrm{O}_{4} \mathrm{Si}\left(\mathrm{MH}^{+}\right)$requires 493.2774 .

(2S,5S,7S,10R)-Spiro[7-(2-tert-butyldiphenylsilanyloxy)ethyl-5-methyl-1,6-dioxabicyclo[4.4.0]decane-2,2'$[2,5]$ dihydrofuran[5]one] (20)

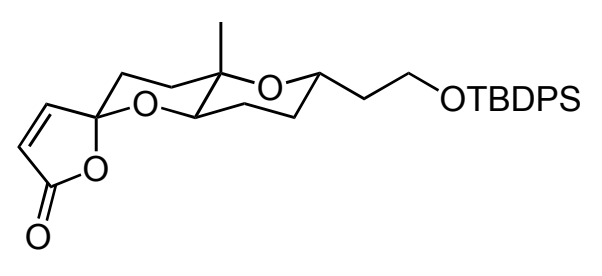

To a stirred solution of alcohol $19(40 \mathrm{mg}, 0.08 \mathrm{mmol})$ in anhydrous dichloromethane $(3 \mathrm{~mL})$ at $0{ }^{\circ} \mathrm{C}$ was added MCPBA (22 mg, $0.09 \mathrm{mmol}, 70 \% \mathrm{wt}$ ) and the reaction mixture was left to stir at $0{ }^{\circ} \mathrm{C}$ for 2 h. The reaction was quenched by the addition of $50 \%$ sodium thiosulfate solution ( $4 \mathrm{~mL})$ and $50 \%$ sodium hydrogen carbonate solution $(4 \mathrm{~mL})$ and was then extracted with ethyl acetate $(4 \square 10 \mathrm{~mL})$. The combined organic extracts were washed successively with brine $(3 \square 20 \mathrm{~mL})$, dried $\left(\mathrm{MgSO}_{4}\right)$, filtered, and the solvent removed in vacuo. The residue was subjected to column chromatography using silica gel (ether/petrol, 3:1) to afford the intermediate lactol (35 mg, $85 \%$ ) as a colourless oil. This lactol (30 $\mathrm{mg}, 0.059 \mathrm{mmol})$ and $N$-methylmorpholine- $N$-oxide $(8.0 \mathrm{mg}, 0.068 \mathrm{mmol})$ were dissolved in anhydrous dichloromethane $(2 \mathrm{~mL})$ and allowed to stir at room temperature for $10 \mathrm{~min}$. Tetrapropylammonium perruthenate $(2.0 \mathrm{mg}, 0.057 \mathrm{mmol})$ was added and the mixture left to stir at room temperature for $3 \mathrm{~h}$. The reaction mixture was filtered through a short plug of silica and the filtrate concentrated in vacuo. The residue was subjected to column chromatography using silica gel (ether/petrol, 3:1) to afford to afford spirobutenolide $20(27 \mathrm{mg}, 90 \%)$ as a pale yellow oil. [ $\square]_{\mathrm{D}}^{22}+8.0\left(c 1.25, \mathrm{CHCl}_{3}\right) ; \square_{\max } / \mathrm{cm}^{-1}$ (thin film) 2928s, 2860s, 1775s, 1428m, 1261s, 1111s, 912m, 801s, 703s; 口н (500 MHz, $\left.\mathrm{CDCl}_{3}\right) 1.06$ $(9 \mathrm{H}, \mathrm{s}, t-\mathrm{Bu}), 1.27(3 \mathrm{H}, \mathrm{s}, \mathrm{Me}), 1.36-1.48(1 \mathrm{H}, \mathrm{m}, \mathrm{H}-8), 1.64-1.71(5 \mathrm{H}, \mathrm{m}, 2 \square \mathrm{H} 9, \mathrm{H}-8$, 
$\left.\mathrm{CH}_{2} \mathrm{CH}_{2} \mathrm{OTBDPS}\right), 1.76$ (1H, ddd, $J$ 13.2, 5.5, $\left.2.5 \mathrm{~Hz}, \mathrm{H}-4\right), 1.82-1.88$ (1H, m, H-3), 1.97 (1H, td, $J$ 13.2, 4.6 Hz, H-4), 2.12 (1H, td, $J 13.2,5.5 \mathrm{~Hz}, \mathrm{H}-3), 3.69(1 \mathrm{H}, \mathrm{dt}, J 10.6,5.3 \mathrm{~Hz})$ and 3.79-3.85 (1H, m, $\left.\mathrm{CH}_{2} \mathrm{OTBDPS}\right), 3.89$ (1H, dd, $J$ 12.0, $\left.4.4 \mathrm{~Hz}, \mathrm{H}-10\right), 3.93-3.99$ (1H, m, H-7), 6.14 (1H, d, J 5.6 Hz, H-4'), 7.12 (1H, d, J 5.6 Hz, H-3'), 7.37-7.45 (6H, m, Ph), 7.66-7.69 (4H, m, Ph); 口c (125 MHz, $\left.\mathrm{CDCl}_{3}\right) 13.7(\mathrm{Me}), 19.1(\mathrm{C}), 24.1\left(\mathrm{CH}_{2}\right), 26.7\left(\mathrm{CH}_{3}\right), 30.9\left(\mathrm{CH}_{2}\right), 31.7\left(\mathrm{CH}_{2}\right), 34.2\left(\mathrm{CH}_{2}\right), 38.9\left(\mathrm{CH}_{2}\right)$, $60.1\left(\mathrm{CH}_{2}\right), 66.0(\mathrm{CH}), 70.4(\mathrm{C}), c a .77 .0\left(\mathrm{CH}\right.$, obscured by $\mathrm{CDCl}_{3}$ resonance), $106.7(\mathrm{C}), 123.3(\mathrm{CH})$, $127.5(\mathrm{CH}), 129.4(\mathrm{Ph}), 133.8(\mathrm{C}), 135.4(\mathrm{CH}), 153.5(\mathrm{CH}), 170.1(\mathrm{C})$; HRMS found 507.2573; $\mathrm{C}_{30} \mathrm{H}_{39} \mathrm{O}_{4} \mathrm{Si}\left(\mathrm{MH}^{+}\right)$requires 507.2567. 